\title{
AN ITERATION TECHNIQUE AND COMMUTATIVITY OF RINGS
}

\author{
H.A.S. ABUJABAL \\ and \\ M.S. KHAN \\ Department of Mathematics \\ Faculty of Science \\ King Abdul Aziz University \\ P.0. BOX 9028 \\ Jeddah-21413 \\ Saudi Arabia \\ (Received June 6, 1988)
}

ABSTRACT. Through much shorter proofs, some new communtativity theorems for rings with unity have been obtained. These results either extend or generalize a few wellknown theorems. Our method of proof is based on an iteration technique.

KEY WORDS AND PHRASES. Communtative rings, torsion free rings. 1980 AMS SUBJECT CLASSIFICATION CODE. Prima ry 16 A70

1. INTRODUCTION.

Recently, Psomopoulos [1], Xuan [2], and Harmanc1 [3] proved some interesting theorems on the commutativity of rings. Our objective in this paper is to present some new commutativity theorems for rings with unity using an iteration type technique developed by Tong [4].

Throughout the rest of the paper, $R$ stands for an associative ring with unity 1. As usua $1,[x, y]=x y-y x$. The following results will be frequently used in the sequel.

LEMMA 1.1. (Nicholson and Yaqub [5]). Let $R$ be a ring with unity 1 and $f: R \rightarrow R$ be a function such that $f(x+1)=f(x)$ for every $x$ in $R$. If for some positive interger $n, x^{n} f(x)=0$ for all $x$ in $R$, then $f(x)=0$.

LEMMA 1.2. (Tong [4]). Let $R$ be a ring with unity 1 . Let $I_{0}^{r}(x)=x^{r}$. If $k>1$, let $I_{k}^{r}(x)=I_{k-1}^{r}(x+1)-I_{k-1}^{r}(x)$. Then $I_{r-1}^{r}(x)=\frac{1}{2}(r-1) r !+r ! x$; $I_{r}^{r}(x)=r$ ! , and $I_{j}^{r}(x)=0$ for $j>r$.

2. RESULTS.

A generalization of a famous result due to Bell [6] was obtained by Psomopoulos [1] through a tedious proof. Here we present an entirely different but easier and 
shorter proof of a similar result.

THEOREM 2.1. Let $n(>1)$ and $m$ be two fixed positive integers and $t, s$ be any nonnegative integers. Let $R$ be an associative ring with unity. Suppose that $x^{t}\left[x^{n}, y\right]=\left[x, y^{m}\right] x^{s}$ for all $x, y$ in $R$. If, further, $R$ is $m !-t o r s i o n$ free, then $R$ is commutative.

PRO0F. Following Tong [4], we set $I_{j}(y)=I_{j}^{m}(y)$, for $j=0,1,2, \ldots$ Then we have

$$
x^{t}\left[x^{n}, y\right]=\left[x, I_{0}(y)\right] x^{s}
$$

Put $y=1+y$ in the above expression to get

$$
x^{t}\left[x^{n}, y\right]=\left[x, I_{0}(1+y)\right] x^{s}
$$

In view of Lemma 1.2 , we get

$$
x^{t}\left[x^{n}, y\right]=\left[x, I_{1}(y)+I_{0}(y)\right] x^{s} \text {. }
$$

Now combining the last identity with (2.1) we are left with

$$
\left[x, I_{1}(y)\right] x^{s}=0 \text {. }
$$

Further, replacing $y$ by $1+y$ in (2.3) and again using Lemma 1.2 , we obtain

$$
\left[x, I_{2}(y)\right] x^{s}=0 \text {. }
$$

Let $y=1+y$ in $(2.4)$. Then we get

$$
\left[x, I_{3}(y)\right] x^{s}=0
$$

Again, replacing $y$ by $1+y$ in the above identity and then iterating $m-1$ times, we obtain the following

$$
\left[x, I_{m-1}(y)\right] x^{s}=0
$$

An application of Lemma 1.2 now yields

$$
\mathrm{m} ![y, x] \mathrm{x}^{\mathrm{s}}=0 \text {. }
$$

By the hypothesis of the theorem, and the Lemma 1.1, the commutativity of $R$ is obvious. This completes the proof.

Our first Corollary resembles Theorem 5 of Bell [6].

COROLLARY 1. Let $R$ be a ring with unity and $n>1$, $m$ be fixed positive

integers. If the identity $\left[x^{n}, y\right]=\left[x, y^{m}\right]$ holds for $x, y$ in $R$, and further $R$ is $m !-$ torsion free, then $R$ is commutative.

COROLLARY 2. (Psomopoulos et a1 [7]).

Let $n>m>1$ be fixed integers with $m>1$, and let $R$ be an s-unital ring. Suppose that every commutator in $R$ is $m$ !- torsion free. If, further, $R$ satisfies the polynomial identity $\left[\mathrm{x}^{\mathrm{n}}, \mathrm{y}\right]=\left[\mathrm{x}, \mathrm{y}^{\mathrm{m}}\right]$, then $\mathrm{R}$ is commutative. More recently, generalizing a result of Wei Zong Xuan [2], Quadri et al [8] proved that a semi prime ring $R$ in which either (i) $\left[x^{m} y^{n}-x y^{n} x, y\right]=0$ or $\left[x^{m} y^{n}-x y^{n} x, x\right]=0$ holds for all $x, y$ in $R$, is necessarily commutative. Our next result is indeed motivated by the above commutativity conditions. 
THEOREM 2.2. Let $R$ be a ring with identity in which any one of the following properties hold for all $x, y$ in $R$ :

$\left(P_{1}\right)$ : there exists positive integers $m>1$ and $n>1$ such that

$$
\left[x^{m} y^{n}-y x^{m} y, y\right]=0
$$

$\left(\mathrm{P}_{2}\right)$ : there exists positive integers $m>1$ and $n>1$ such that

$$
\left[x^{m} y^{n}-y x^{m} y, x\right]=0 \text {. }
$$

If $R$ contains no nonzero element $x$ for which $n$ ! $m$ ! $x=0$, then $R$ must be commutative.

PROOF. Let us pause to notice that for each $x, y \in R$, the property $\left(P_{1}\right)$ is equivalent to

$$
\left[x^{m}, y\right] y^{n}=y\left[x^{m}, y\right] y \cdot
$$

Let $I_{j}(y)=I_{j}^{n}(y), j=0,1,2, \ldots$ Then by Lemma $1.2 I_{0}(y)=y^{n}$ and we have

$$
\left[\mathrm{x}^{\mathrm{m}}, \mathrm{y}\right] \mathrm{I}_{0}(\mathrm{y})=\mathrm{y}\left[\mathrm{x}^{\mathrm{m}}, \mathrm{y}\right] \mathrm{y} \text {. }
$$

Replacing $y$ by $1+y$ in the above identity ylelds

$$
\left[\mathrm{x}^{\mathrm{m}}, 1+\mathrm{y}\right] \mathrm{I}_{0}(1+\mathrm{y})=(1+\mathrm{y})\left[\mathrm{x}^{\mathrm{m}}, 1+\mathrm{y}\right](1+\mathrm{y}) \text {. }
$$

But $I_{0}(1+y)=I_{1}(y)+I_{0}(y)$, by Lemma 1.2 . Hence

$$
\left[x^{m}, y\right] I_{1}(y)=\left[x^{m}, y\right]+y\left[x^{m}, y\right]+\left[x^{m}, y\right] y \bullet
$$

Let $y=1+y$ in $(2.9)$. Then by Lemma 1.2 , we have

$$
\left[x^{m}, y\right] \quad I_{2}(y)=2\left[x^{m}, y\right]
$$

Setting $y=1+y$ in the above identity we get

$$
\left[x^{m}, y\right] I_{3}(y)=0 \text {. }
$$

Similarly, we can get

$$
\left[x^{m}, y\right] I_{4}(y)=0 \text {. }
$$

By setting $y=1+y$ and iterating (2.11) $n$ times we get

$$
\left[x^{m}, y\right] I_{n}(y)=0 \text {. }
$$

In view of Lemma 1.2 , the above identity gives

$$
n !\left[x^{m}, y\right]=0 \text {. }
$$

But since $R$ is $n$ !-torsion free, we have

$$
\left[x^{m}, y\right]=0 \text {. }
$$

Now we shall apply iteration to $\mathrm{x}^{\mathrm{m}}$.

Put $x^{m}=I_{0}(x)$, and then set $x=1+x$ in (2.13) which is then reduced to

$$
\left[I_{0}(1+x), y\right]=0 \text {. }
$$

Using Lemma 1.2 and iterating the above identity m-l times, we get

$$
\mathrm{m} ![\mathrm{x}, \mathrm{y}]=0 \text {. }
$$


Since every commutator in $R$ is $m$ !-torsion free, we get $[x, y]=0$, which implies the commutativity of $R$. Now, suppose that $R$ satisfies $\left[x^{m} y^{n}-y x^{m} y, x\right]=0$. As above, we first take $I_{0}(y)=y^{n}$, and then replace $y$ by $1+y$. Thereafter iterating $n-1$ times we get

$$
\mathrm{n} !\left[\mathrm{x}^{\mathrm{m}} \mathrm{y}, \mathrm{x}\right]=0
$$

Set $x^{m}=I_{0}(x)$ in $(2.14)$. Replace $x$ by $1+x$ and iterate $m$ times to reach

$$
n ! m ![x, y]=0 .
$$

Thus $R$ is commutative.

REMARK. From (2.14), the torsion condition on $R$ implies that $\left[x^{m} y, x\right]=x^{m}[y, x]=0$. Hence by Lemma 1.1 , we immediately see that $R$ is commutative. Thus, in fact we only require $R$ to be $n$ !-torsion free when it satisfies $\left(\mathrm{P}_{2}\right)$.

The next result seems to be new in its own right.

THEOREM 2.3. Let $R$ be a ring with unity. If for all $x, y$ in $R$ any one of the following properties holds

$$
\begin{array}{ll}
\left(Q_{1}\right): & {\left[\left(x^{n}-y^{m}\right) x y, y\right]=0,} \\
\left(Q_{2}\right): & {\left[\left(x^{n}-y^{m}\right) x y, x\right]=0,}
\end{array}
$$

then $R$ is necessarily commutative provided it contains no non-zero element $x$ for which $(n+1) ! x=0$.

PROOF. First, let $R$ satisfy $\left(Q_{1}\right)$. Clearly $\left(Q_{1}\right)$ can be rewritten as

$$
\left[x^{n+1}, y\right] y=y^{m}[x, y] y \cdot
$$

Let $I_{0}(x)=x^{n+1}$ in the equation (2.15). Replace $x$ by $1+x$ in (2.15) and iterate it $n$ times. Then following the idea of proof as in our earlier results, we get

$$
(n+1) ![x, y]=0 \text {, }
$$

which clearly forces $R$ to be commutative. In case, $R$ satisfies $\left(Q_{2}\right)$, we can write it in the form

$$
\left[x^{n+1} y, x\right]=\left[y^{m} x y, x\right]
$$

We let $I_{0}(x)=x^{n+1}$ in (2.16). As above, set $x=1+x$ and iterate $n+1$ times to reach the identity

$$
(n+1) ![y, x]=0,
$$

which implies the commutativity of $R$. This completes the proof.

Our last result is motivated by the polynomial identity considered by Harmanci [3].

THEOREM 2.4. Let $R$ be a ring with unity and $m>1, n>1$ be two fixed integers such that the identity 


$$
x^{m}\left[x, y^{n}\right] y=0
$$

holds for all $x, y$, in $R$. If the characteristic of $R$ does not divide $n(m !)(n !)$, then $R$ is commutative.

PROOF. Let $I_{j}(y)=I_{j}^{n}(y)$, for $j=0,1,2, \ldots$ Then we have

$$
x^{m}\left[x, I_{0}(y)\right] y=0
$$

Replacing $y$ by $1+y$ in (2.18) and then iterating $n-1$ times, we get

$$
n(n !) x^{m}[x, y]=0 \text {. }
$$

Now put $x^{m}=I_{0}(x)$. Then after letting $x=1+x$ in the above expression, $m$ iterations yield

$$
n(m !)(n !)[x, y]=0,
$$

whence $R$ is clearly commutative. This ends the proof.

REMARKS. (1) For $m=n$, Theorem 2.4 is reduced to Theorem A of Harmanc1 [3] • However, our proof is much shorter and does not use any combinatorial type arguments. (11) As in the remark given after Theorem 2.2, Lemma 1.1 can very well be applied to conclude the commutativity of $R$ when $i t$ is assumed to be only $n(n !)$-torsion free. Thus in this way we get a slight improvement over the result of Harmanc1 [3].

ACKNOWLEDGEMENT. We are grateful to Professor E. Psomopoulos and Dr. M.A. Khan for providing us with reprints of their papers which have motivatied the present study.

\section{REFERENCES}

1. PSOMOPOULOS, E., Commutativity theorms for rings and groups constraints on commutators, Internat. J. Math. Math. Sci., 7(3) (1984), 513-517.

2. XUAN, W., A note on the commutativity of Semi-prime rings, J. Math. Res . Exposition, 5 (1985), 109-110.

3. HARMANCI, A., Two elementary commutativity theorems for rings, Acta Math. Acad. Sc1. Hunga r. 29 (1977), 23-29.

4. TONG, J., On the commutativity of a ring with identity, Canad. Math. Bull., 27(4) (1984), 456-460.

5. NICHOLSON, W.K. and YAQUB, A., A commutativity theorem for rings and groups, Canad. Math. Bul1., 22 (1979), 419-423.

6. BELL, H.E., On the power map and ring commutativity, Canad. Math. Bul1., 21 (1978), 399-404.

7. PSOMOPOULOS, E., TOMINAGA, H. and YAQUB, A., Some commutativity theorems for n-torsion free rings, Math. J. Okayama Univ., 23 (1981), 37-39.

8. QUADRI, M.A., KHAN, M.A. and ASMA A., On a commutativity condition for rings, (pre-print). 


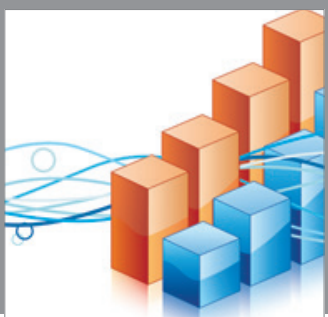

Advances in

Operations Research

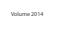

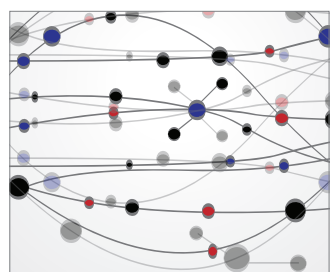

\section{The Scientific} World Journal
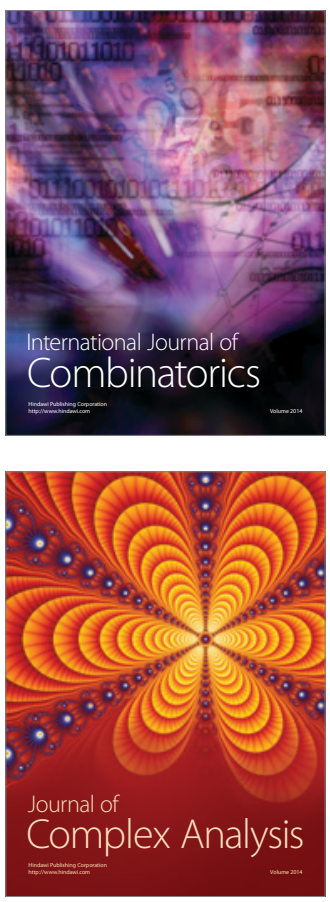

International Journal of

Mathematics and

Mathematical

Sciences
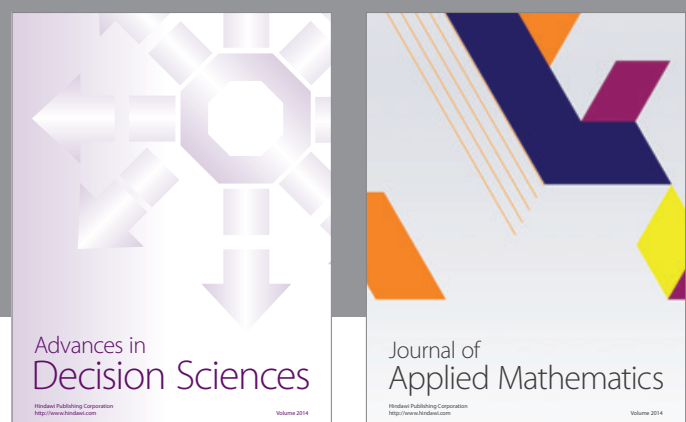

Journal of

Applied Mathematics
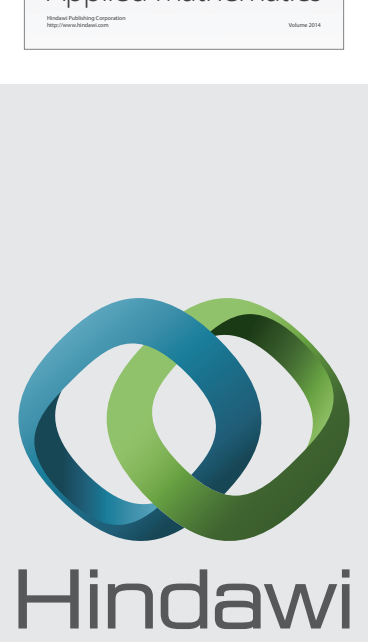

Submit your manuscripts at http://www.hindawi.com
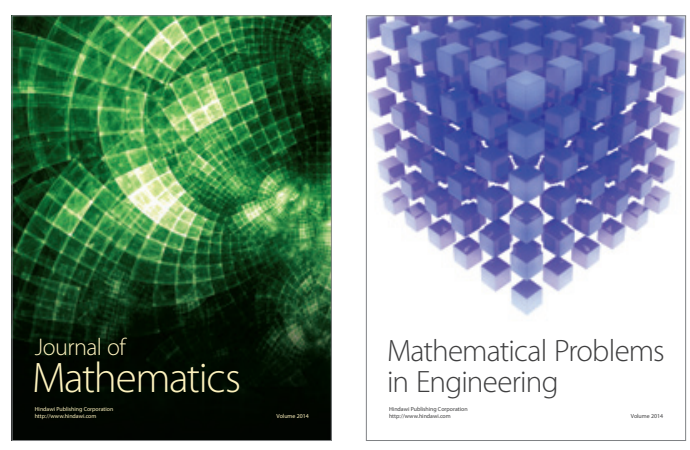

Mathematical Problems in Engineering
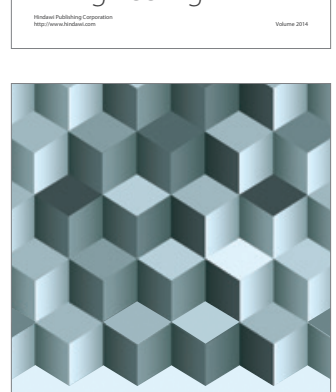

Journal of

Function Spaces
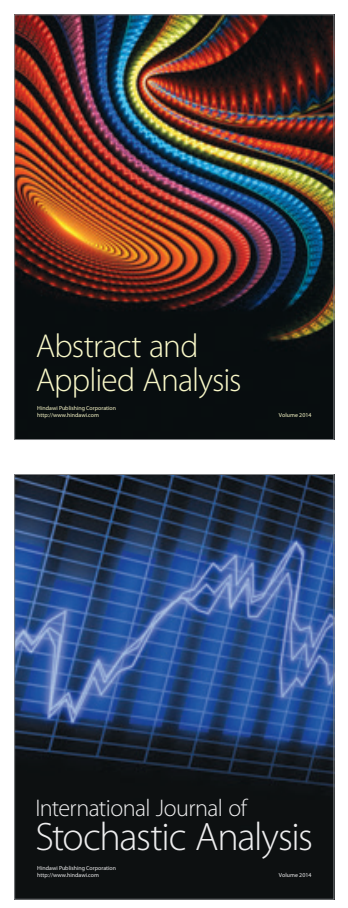

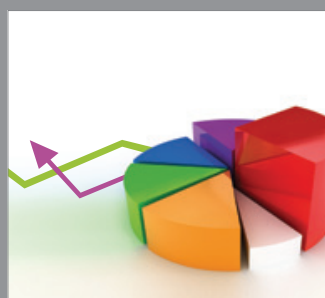

ournal of

Probability and Statistics

Promensencen
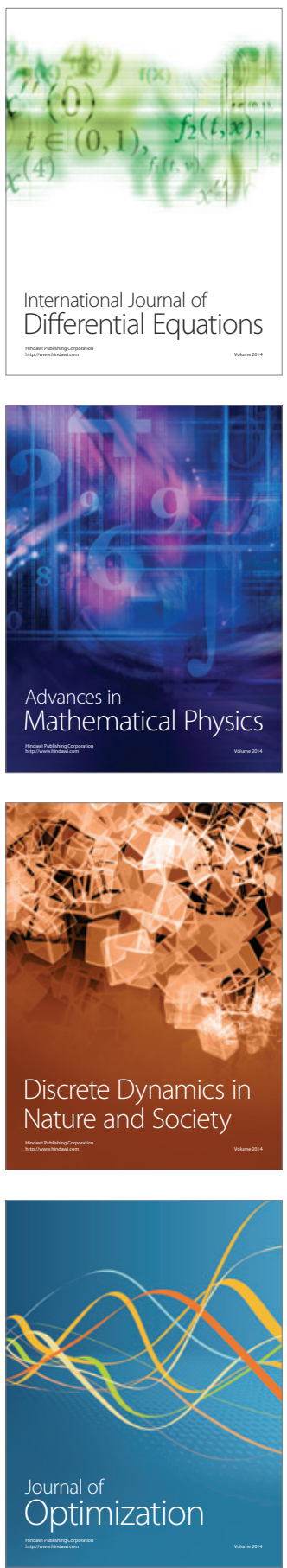